\title{
Recent results from the Pierre Auger Observatory on the mass composition and hadronic interactions of ultra-high energy cosmic rays
}

\author{
Alexey Yushkov $^{1 \mathrm{a}}$ for the Pierre Auger Collaboration ${ }^{2,3}$ \\ ${ }^{1}$ Instituto de Tecnologías en Detección y Astropartículas (CNEA, CONICET, UNSAM), Centro Atómico Constituyentes, Comisión \\ Nacional de Energía Atómica, Argentina \\ 2 Observatorio Pierre Auger, Av. San Martín Norte 304, 5613, Malargüe, Argentina \\ ${ }^{3}$ Full author list: http://www . auger.org/archive/authors_2016_08.html
}

\begin{abstract}
Recent results on the mass composition and hadronic interactions of ultra-high energy cosmic rays (UHECR) obtained at the Pierre Auger Observatory are reviewed. Different studies based on shower observables recorded with the fluorescence and surface detectors of the Observatory indicate that the mass composition is getting lighter for the energies below $\approx 2 \mathrm{EeV}$ and heavier afterwards. A fully consistent description of the data collected at the Observatory cannot be achieved with any of the up-to-date interaction models.
\end{abstract}

\section{Introduction}

A large variety of data, with unprecedented statistics, has been collected at the Pierre Auger Observatory [1] since the start of the operation in 2004. Analysis of the UHECR mass composition can be performed using different characteristics of the longitudinal and lateral profiles of electromagnetic (EM) and muon shower components with the methods having independent systematic uncertainties. The approaches relying on the studies of the EM component (analysis of the depth of the shower maximum $X_{\max }$ ) or using general properties of the extensive air showers (analysis of the correlation between $X_{\max }$ and the signal in surface stations or studies of the elongation rates) have smaller uncertainties due to hadronic interaction models compared to the approaches making use of absolute values related to the muon component. In this proceedings the results on the mass composition from the first and second types of the methods are compared and the conclusions on both the evolution of the primary mass with energy and on the drawbacks of the interaction models (EPOS-LHC [2], QGSJetII-04 [3], Sibyll 2.1 [4]) are presented.

The data reviewed in the paper are recorded using the fluorescence (FD) and surface detectors (SD) of the Pierre Auger Observatory, located in the Mendoza province, Argentina. The SD consists of 1660 water-Cherenkov detectors (WCD) placed in a triangular grid with a spacing of 1500 meters over the area of $3000 \mathrm{~km}^{2}$. Twenty-seven telescopes of the FD are located at four sites on the perimeter of the SD. The FD operates only during clear moonless nights and its duty cycle is around $15 \%$, while the duty cycle of the SD is $\approx 100 \%$. Showers triggering both FD and SD are called hybrid events. For them, complementary information from both detectors allows one to reconstruct the shower parameters with higher

a e-mail: yushkov .alexey@gmail.com precision and to perform cross-checks of the operation of the detectors. Three of the analyses presented below measurements of the depth of shower maximum $X_{\max }$, of the correlation between $X_{\max }$ and signal in WCDs, and of the muon content in highly inclined events - exploit the advantages of the hybrid design of the Observatory. The measurements of risetime asymmetry and of muon production depth are performed using information from WCDs on particle arriving times sampled at the rate of $40 \mathrm{MHz}$.

Unless otherwise specified the data used are from the period from January 2004 till December 2012.

\section{Measurement of the depth of shower maximum $X_{\max }[5,6]$}

Direct determination of $X_{\max }$ and the calorimetric measurement of the energy of the primary particle are based on the detection of fluorescence light emitted by the molecules of atmospheric nitrogen due to their interactions with the shower particles. The parameters of the atmosphere, acting as a calorimeter, are thoroughly monitored at the Observatory and only events passing a pre-selection on good atmospheric conditions (cloud coverage, aerosol density) are accepted for the analysis. These events are subject to further selection ensuring unbiased measurement of the $X_{\max }$ distributions including specific requirements on the field of view of the FD telescopes and the quality cuts on the profile. For full details on the selection and reconstruction procedures the reader is referred to $[5,6]$.

In June 2010 three High Elevation Auger Telescopes (HEAT) started to take data in addition to the standard 24 telescopes of the FD. The HEAT in combination with the standard telescopes at the Coihueco site (called further $\mathrm{HeCo}$ ) have a field of view $2^{\circ}-60^{\circ}$ in elevation (compared to $2^{\circ}-30^{\circ}$ of the standard FD) and in this 

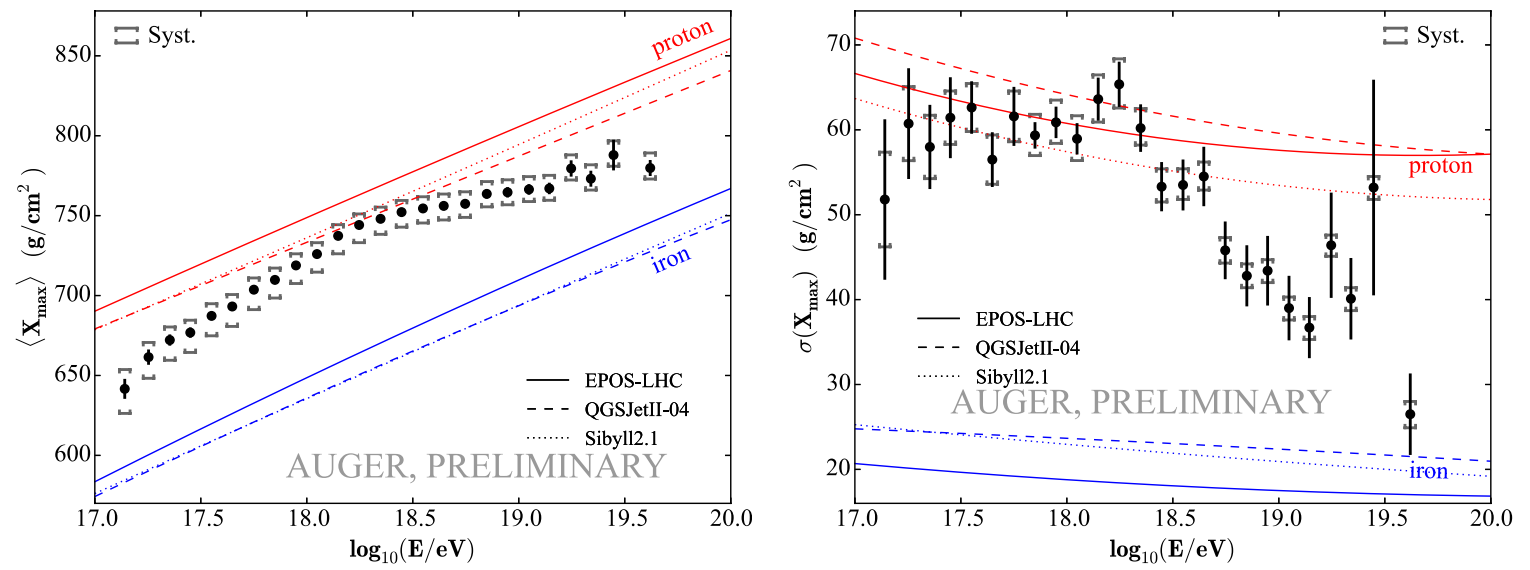

Figure 1. The mean and the standard deviation of the measured $X_{\max }$ distributions compared to the simulations with EPOS-LHC, QGSJetII-04and Sibyll 2.1for protons and iron.
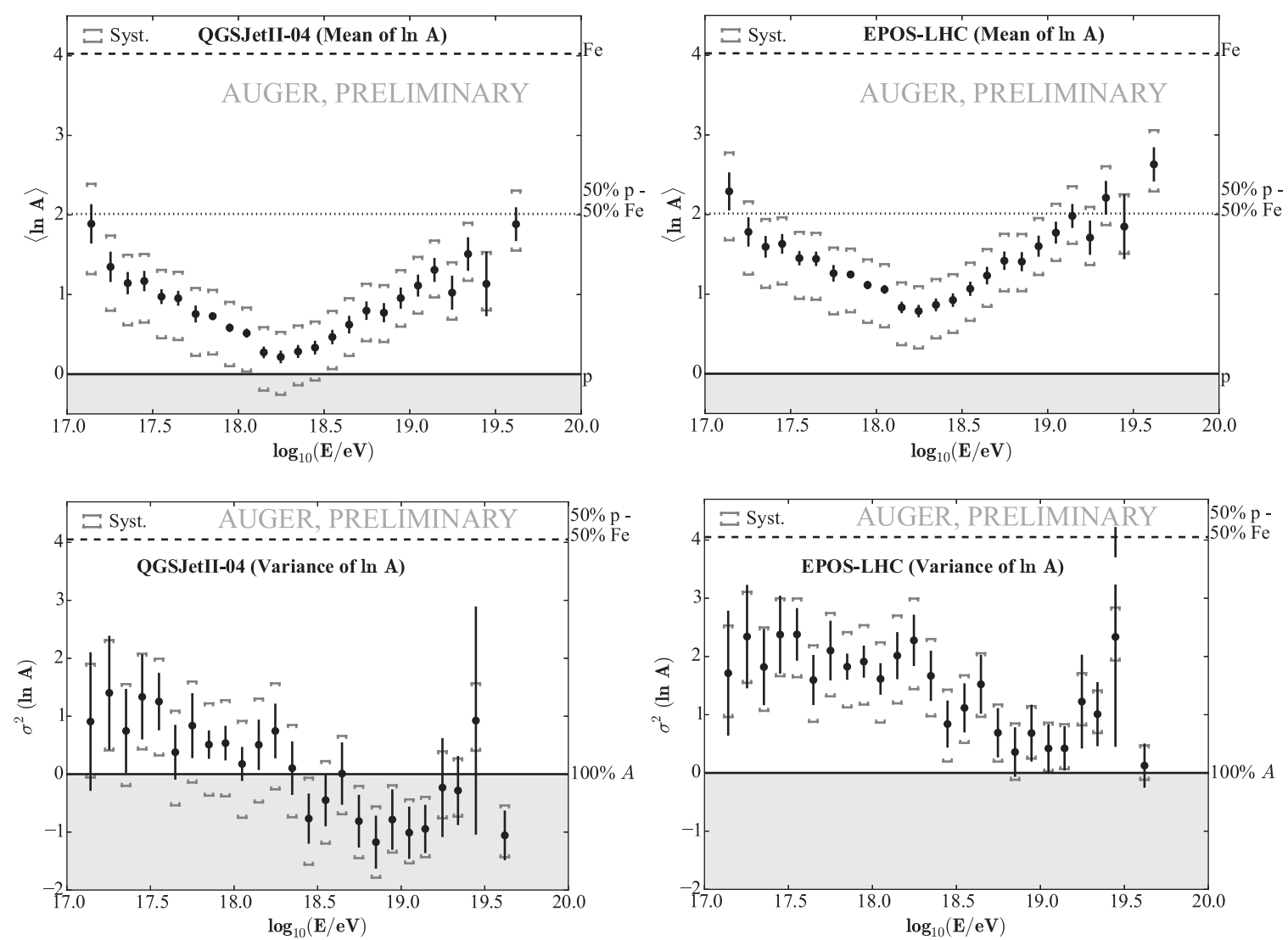

Figure 2. The evolution of the mean (top) and of the variance (bottom) of $\ln A$ determined from the measurements of $X_{\max }$.

way nearby showers with energies down to $10^{17} \mathrm{eV}$ can be observed. To make the data set recorded by $\mathrm{HeCo}$ complementary and independent, events (1377 in total) with energies $<10^{18.3} \mathrm{eV}$ observed by HeCo during the period $01 / 06 / 2010-15 / 08 / 2012$ are excluded from the data of the standard FD.

The final data set contains $5490 \mathrm{HeCo}$ events with energies $10^{17.0}-10^{18.3} \mathrm{eV}$ and 18382 events from the standard FD with energies $>10^{17.8} \mathrm{eV}$. The $X_{\max }$ resolution is around $30 \mathrm{~g} / \mathrm{cm}^{2}$ at $10^{17.0} \mathrm{eV}$ and improves with the energy to $15 \mathrm{~g} / \mathrm{cm}^{2}$ for $E>10^{18.5} \mathrm{eV}$. The systematic uncertainty is below $(-15 ;+10) \mathrm{g} / \mathrm{cm}^{2}$ for most of the energies.

The comparison of the first two central moments of the measured $X_{\max }$ distributions with predictions of different hadronic interaction models for protons and iron nuclei is given in Fig. 1. The observed elongation rate (ER) is described well with a broken line, the energy of the break is $\lg \left(E_{0} / \mathrm{eV}\right) \approx 18.3\left(E_{0} \approx 2 \mathrm{EeV}\right)$ and the slopes below and above the break are equal to $\approx 85$ and $\approx 26\left[\mathrm{~g} / \mathrm{cm}^{2} /\right.$ decade] correspondingly. The ER in simulations for all hadronic interaction models and all constant compositions lies in the range of $54-64\left[\mathrm{~g} / \mathrm{cm}^{2} /\right.$ decade $]$, thus the observed ERs indicate that the primary mass is decreasing below the break around $2 \mathrm{EeV}$ and is increasing for the higher energies. Using the superposition model (see e.g. $[7,8])$ the mean logarithmic mass can be found from the measured $\left\langle X_{\max }\right\rangle$ using the relation $\left\langle X_{\max }\right\rangle \approx\left\langle X_{\max }^{p}\right\rangle-$ $\langle\ln A\rangle D_{10} / \ln (10)$, where $\left\langle X_{\max }^{p}\right\rangle$ is the value for protons. The evolution of $\langle\ln A\rangle$ with energy is shown in Fig. 2 (top row). The observed transition from lighter to heavier 
composition implies that the primary beam is not pure and represents a mix of different nuclei. The degree of mixing (the variance of the logarithmic masses $\sigma_{\ln A}^{2}$ ) can be estimated using the measured width of the $X_{\max }$ distributions [9]: $\sigma^{2}\left(X_{\max }\right) \approx\left\langle\sigma_{\mathrm{sh}}^{2}\right\rangle+\sigma_{\ln A}^{2}\left(D_{10} / \ln (10)\right)^{2}$, where $\sigma_{\mathrm{sh}}$ is the shower-to-shower $X_{\max }$ fluctuations for a fixed mass. The results on $\sigma_{\ln A}^{2}$, presented in Fig. 2 (bottom row), show different degrees of mixing in the primary beam depending on the hadronic interaction model used. Though for QGSJetII-04the variance of the masses takes negative values for some of the energies, the discrepancy is not larger than two standard statistical deviations and is non-significant if one takes into account the systematic errors on $X_{\max }$ measurements and on the FD energy scale.

\section{Measurement of the correlation between $X_{\max }$ and $S(1000)$ [10]}

The spread of the masses in the primary beam $\sigma(\ln A)=\sqrt{\left\langle\ln ^{2} A\right\rangle-\langle\ln A\rangle^{2}}$ can be estimated from the measurement of the correlation between $X_{\max }$ and the signal in WCDs at $1000 \mathrm{~m}$ from the shower axis $S(1000)[10,11]$. In simulations this correlation is close to zero or is slightly positive for pure compositions. Mixed compositions show a negative correlation: heavier primary nuclei have shallower $X_{\max }\left(\Delta X_{\max } \sim-\Delta \ln A\right)$ and larger $S(1000)$ (due to a larger number of muons $\left.N_{\mu} \sim A^{1-\beta}, \beta \simeq 0.9[12]\right)$, thus in a sample with a mixed composition the shallower showers on average will have larger signals. Such behavior is a general property of extensive air showers and thus the results of the correlation analysis are weakly sensitive to the choice of a particular interaction model.

In this analysis, to avoid a decorrelation due to the spreads of energies and zenith angles, $X_{\max }$ and $S(1000)$ are scaled to a reference energy of $10 \mathrm{EeV}$ and $S(1000)$ is additionally scaled to zenith angle of $38^{\circ}$. The scaled variables are denoted further as $X_{\max }^{*}$ and $S_{38}^{*}$. The correlation between $X_{\max }^{*}$ and $S_{38}^{*}$ is evaluated using a ranking correlation coefficient $r_{\mathrm{G}}$ proposed in [13]. The conclusions do not change when other correlation coefficients are used.

To have a data set with an unbiased mass composition the event selection is the same as in the $X_{\max }$ analysis [6]. In addition, to have a reliable reconstruction of $S(1000)$, it is required that the SD station with the highest signal should have at least 5 active neighbour stations. For the energy range $\lg (E / \mathrm{eV})=18.5-19.0$ and the zenith angle range $0^{\circ}-65^{\circ}$, the final data set contains $n=1376$ events. The statistical uncertainty can be approximated [10] by $\Delta r_{\mathrm{G}} \simeq$ $0.9 / \sqrt{n}$, thus $\Delta r_{\mathrm{G}}($ data $)=0.024$. The systematic error on $r_{\mathrm{G}}$ is small $\lesssim 0.01$ : invariance of the ranking correlation coefficients to any transformations leaving ranks of the events unchanged makes the correlation analysis almost insensitive to the systematic uncertainties in $X_{\max }$ and $S(1000)$.

In Fig. 3 the distributions of $X_{\max }^{*}$ and $S_{38}^{*}$ in data and in simulations for protons and iron with EPOS-LHCare shown together with the corresponding values of the correlation coefficients. The correlation coefficients for protons for all models are non-negative: $r_{\mathrm{G}}(\mathrm{EPOS}-\mathrm{LHC})=$ $0.00, r_{\mathrm{G}}\left(\right.$ QGSJetII-04) $=0.08$ and $r_{\mathrm{G}}($ Sibyll 2.1$)=0.06$. For heavier nuclei the correlation coefficients are larger
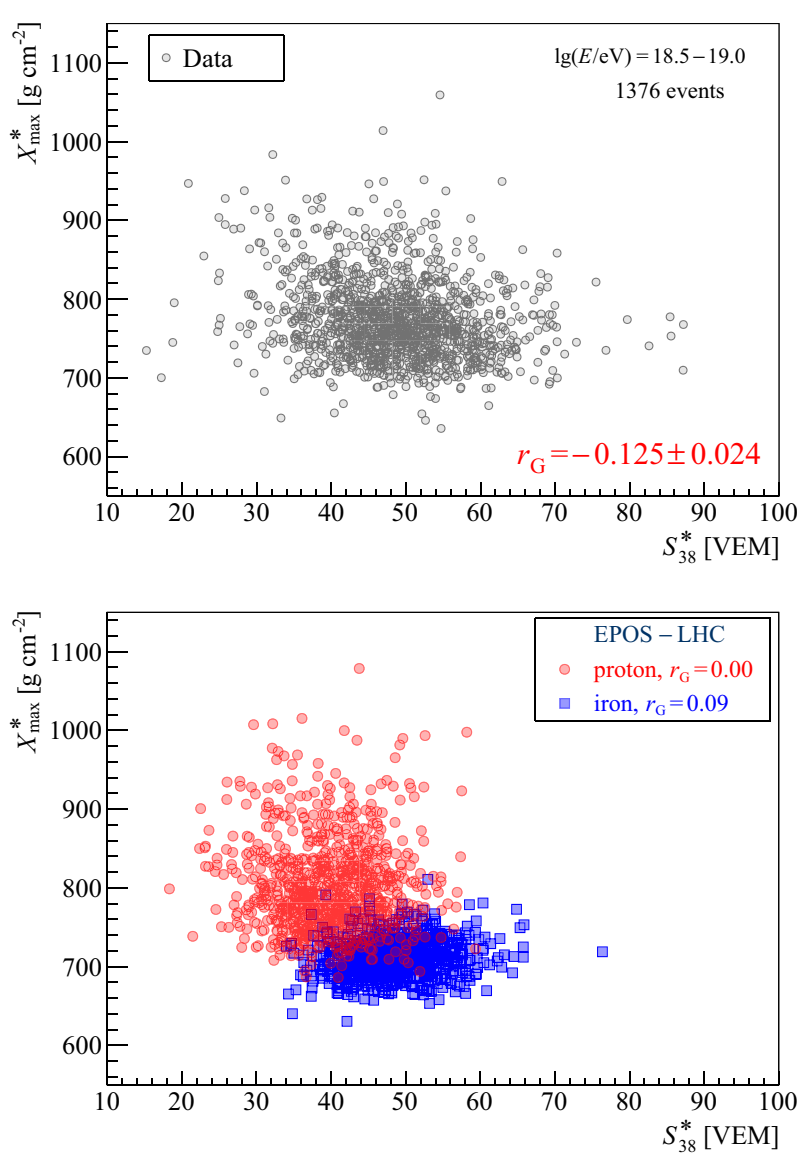

Figure 3. Distributions of $X_{\max }^{*}$ vs. $S_{38}^{*}$ (see text) for $\lg (E / \mathrm{eV})=$ 18.5-19.0. Top: data; bottom: 1000 proton and 1000 iron showers simulated with EPOS-LHC.

than those for protons and for all $p-$ He mixtures $r_{\mathrm{G}} \gtrsim 0$ as well. Thus, the negative correlation found in data $r_{\mathrm{G}}\left(X_{\text {max }}^{*}, S_{38}^{*}\right)=-0.125 \pm 0.024$ (stat) cannot be reproduced using any pure composition and the data can be explained only by mixed compositions containing primary nuclei heavier than helium $A>4$. The conclusions do not change when the key parameters of the interaction models (cross-section, multiplicity, elasticity, pion charge ratio) are modified by a factor $\approx 1.5$.

The spread of the primary masses $\sigma(\ln A)$ can be estimated from Fig. 4 where the correlation value found in data is compared to the values in simulated mixtures with different fractions of protons, helium, oxygen and iron nuclei. The correlation $r_{\mathrm{G}}\left(X_{\max }^{*}, S_{38}^{*}\right)$ gets more negative for the larger spreads of the masses. For all three models (results for Sibyll 2.1, not shown in Fig. 4, are almost the same as for QGSJetII-04) the spread of masses corresponding to the correlation found in data, lies in the range $1.0 \lesssim \sigma(\ln A) \lesssim 1.7$

\section{Azimuthal asymmetry in the risetime of signals in WCDs [14]}

Muons produce short and spiky time traces in WCDs, while for EM particles the distribution of the arrival times is larger and smoother. Since the relative contribution of muons to the SD signal increases with the growth of the mass of the primary nuclei, the risetime, $t_{1 / 2}$, defined as the 

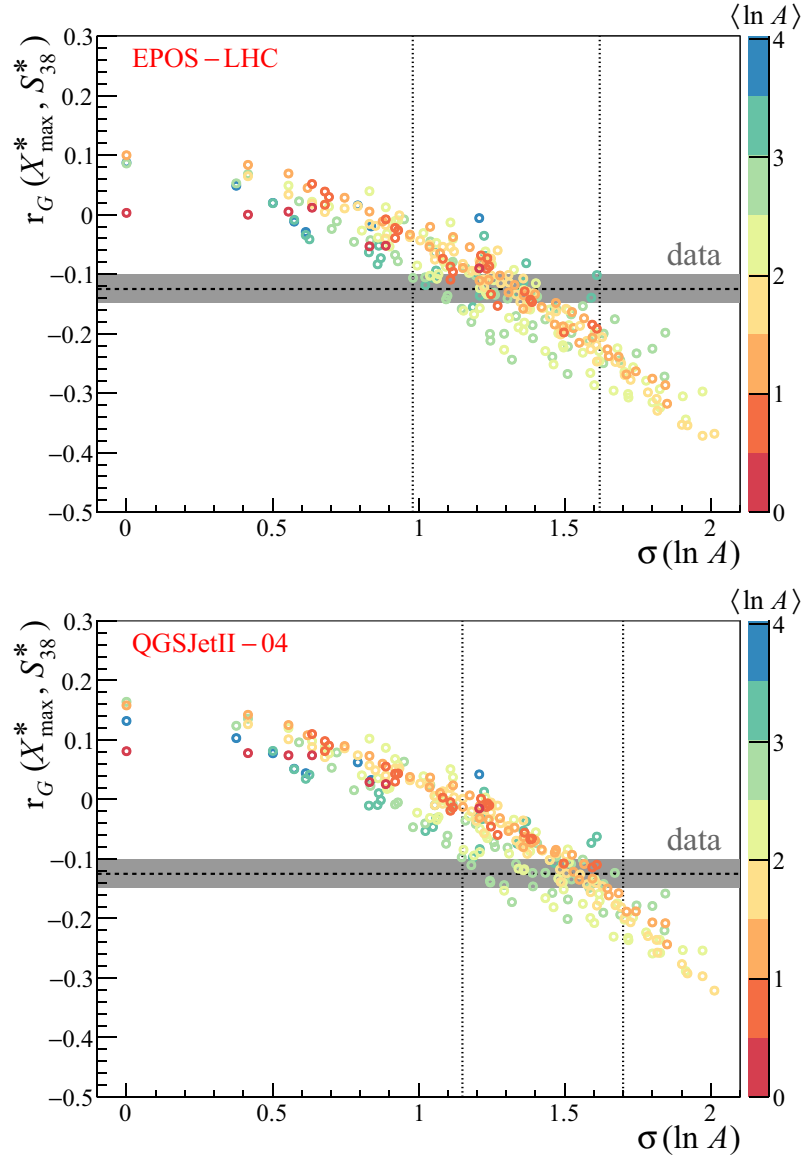

Figure 4. Dependence of the correlation coefficients $r_{\mathrm{G}}$ on $\sigma(\ln A)$ for EPOS-LHC (top) and QGSJetII-04(bottom) in mixtures of protons, helium, oxygen and iron nuclei. The shaded area shows the value for the data $r_{\mathrm{G}}\left(X_{\max }^{*}, S_{38}^{*}\right)=-0.125 \pm$ 0.024 (stat). $\lg (E / \mathrm{eV})=18.5-19.0$.

time of the increase of the total integrated signal in WCDs from $10 \%$ to $50 \%$, is a mass-sensitive observable. Due to changes in the muon/EM signal ratio and in the thickness of the shower front with distance to the shower axis $r$ and zenith angle $\theta$, risetime is a function of $r$ and $\theta$. Risetime grows almost linearly $t_{1 / 2}(r) \propto r$ for a large range of $r$, thus $t_{1 / 2} / r$ is used to cancel out the distance dependence and to include WCDs from all distances in the analysis.

In inclined showers signal risetime in individual WCDs depends on their azimuth angle $\zeta$ in the shower plane: particles hitting 'early' stations $(|\zeta|<\pi / 2)$ cross smaller atmosphere thickness and have smaller angle to the shower axis compared to the particles hitting 'late' stations $(|\zeta|>\pi / 2)$. With the growth of zenith angle fewer EM particles reach late detectors due to absorption by the additional atmosphere (the signals thus become more muon-rich) and risetime in the late detectors becomes shorter compared to that in early detectors. As shown in Fig. 5 (top) the asymmetry in risetimes reaches its maximum at a certain zenith angle and decreases for larger angles where the muon component starts to give a dominant contribution to the signals.

The dependence of the risetime on azimuth angle can be parameterized as $\left\langle t_{1 / 2} / r\right\rangle(\zeta)=a+b \cos \zeta+$ $c \cos ^{2} \zeta$ (Fig. 5 (top)) and the risetime asymmetry can be characterized using the parameter $b /(a+c) \sim$ $\left(\left\langle t_{1 / 2} / r\right\rangle(0)-\left\langle t_{1 / 2} / r\right\rangle(\pi)\right) /\left(\left\langle t_{1 / 2} / r\right\rangle(0)+\left\langle t_{1 / 2} / r\right\rangle(\pi)\right)$.
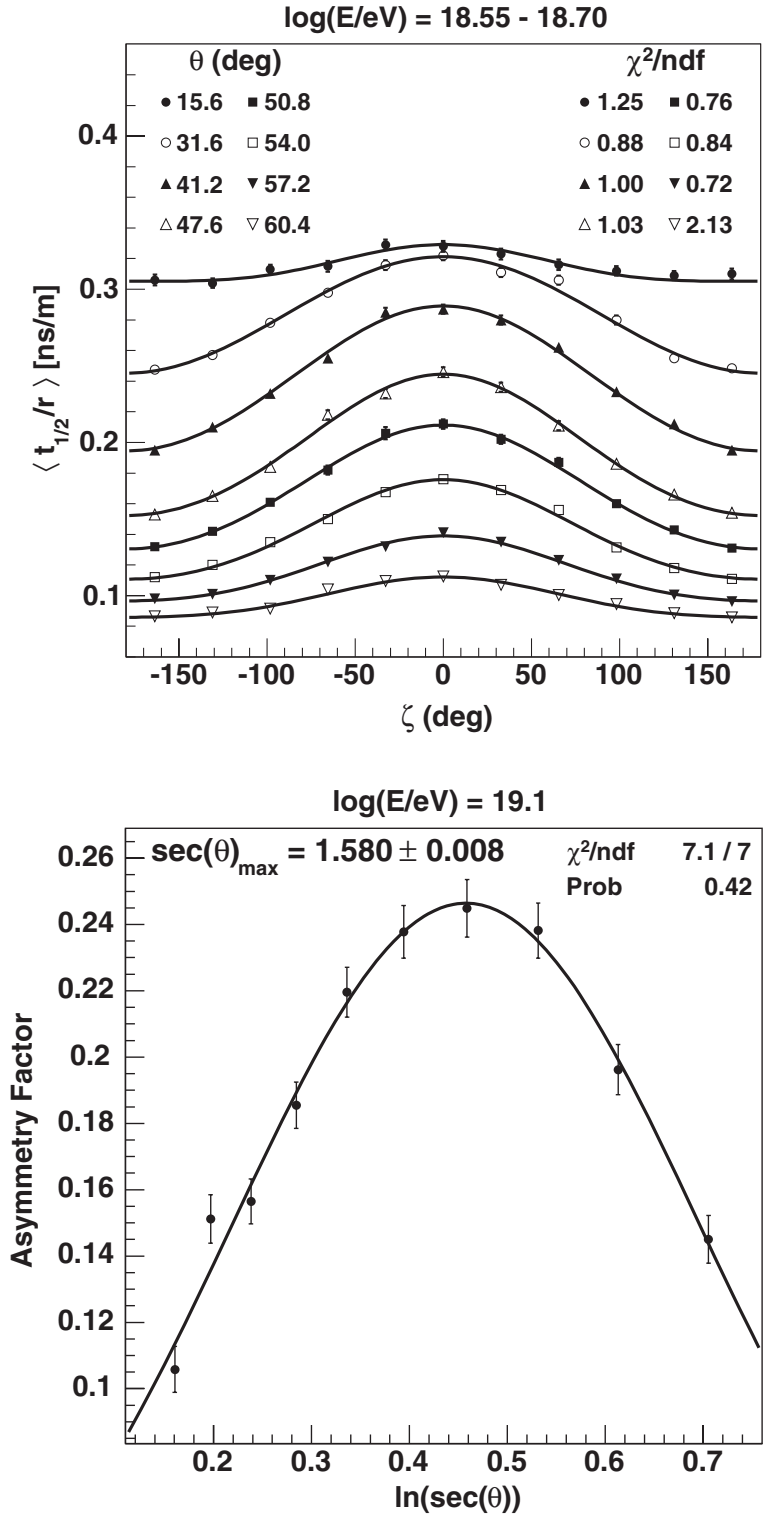

Figure 5. Top: dependence of $\left\langle t_{1 / 2} / r\right\rangle$ on the azimuth angle $\zeta$ fitted with a quadratic function (lines) for various zenith angles $\theta \cdot \lg (E / \mathrm{eV})=18.55-18.70$. Bottom: dependence of the asymmetry factor $b /(a+c)$ on $\ln (\sec (\theta))$ fitted with a Gaussian (line). $\lg (E / \mathrm{eV})=19.1$.

The behavior of the asymmetry parameter with $\ln (\sec (\theta))$ at $\lg (E / \mathrm{eV})=19.1$ in stations within the range $r=500-$ $1000 \mathrm{~m}$ is shown in Fig. 5 (bottom) together with a fit with a Gaussian function. The value of $\sec (\theta)_{\max }$ at which the asymmetry parameter reaches its maximum provides information on the mass of the primary particle.

The behavior of $\sec (\theta)_{\max }$ in the SD data is studied using events with the energies corresponding to $100 \%$ SD efficiency $E>3 \times 10^{18} \mathrm{eV}$ for the zenith angle range of $\theta=30^{\circ}-62^{\circ}$. For an accurate reconstruction of $t_{1 / 2}$ only the stations with the $100 \%$ trigger probability (signal > 10 VEM) are used. The stations should not have saturated traces and the reliable determination of $t_{1 / 2}$ should be possible, these requirements limit the range of the WCDs distances to $r=500-2000 \mathrm{~m}$. For the data recorded from January 2004 to October 2014 a total of 191534 signal traces from 54584 events are selected. The evolution of $\sec (\theta)_{\max }$ with energy is analyzed in two ranges of the 
distances to the shower axis $r=500-1000 \mathrm{~m}$ and $r=$ $1000-2000 \mathrm{~m}$. Systematic error on $\sec (\theta)_{\max }$ is $\approx 0.014$.

The comparison of $\sec (\theta)_{\max }$ in the data and simulations for protons and iron nuclei is shown in Fig. 6. The values for data are bracketed by the QGSJetII-04and EPOS-LHCpredictions for protons and iron. There are indications of an increase of the primary mass with energy, in agreement with other results reported in this proceedings, but uncertainties from the interaction models on the absolute value of $\sec (\theta)_{\max }$ do not allow one to make robust conclusions of average mass of the primary nuclei. For QGSJetII-04such conclusions have an unphysical dependence on the range of the distances to the shower axis favouring lighter composition at larger distances $r=1000-2000 \mathrm{~m}$.

From Fig. 7, where the values of $\sec (\theta)_{\max }$ converted to $\langle\ln A\rangle$ are presented, one can see that the average mass from the risetime asymmetry analysis lies between the values obtained from the measurements of $X_{\max }$ and the muon production depth (MPD, discussed in the next section). This might be due to the different role played by the muon and EM components in each of the analyses: for $X_{\max }$ determination the EM component is of major importance, MPD analysis relies on the reconstruction of properties of muons, and the $\sec (\theta)_{\max }$ behavior results from a complicated interplay between both muon and EM components.

\section{Measurements of the muon production depth $X_{\max }^{\mu}$ [15]}

Muons in the atmosphere travel along almost straight lines and the production height $z$ of each muon along the shower axis can be reconstructed from a quite simple geometry shown in Fig. 8 [15]: $z \approx$ $1 / 2\left(r^{2} / c\left(t-\left\langle t_{\varepsilon}\right\rangle\right)-c\left(t-\left\langle t_{\varepsilon}\right\rangle\right)\right)+\Delta-\left\langle z_{\pi}\right\rangle$, here $\left\langle t_{\varepsilon}\right\rangle-$ mean kinematic delay; $\left\langle z_{\pi}\right\rangle$ - pion decay length. After conversion of heights $z$ to the mass units $X^{\mu}=$ $\int_{z}^{\infty} \rho\left(z^{\prime}\right) d z^{\prime}$ one gets a distribution of the MPD for an event and the depth of the maximum muon production $X_{\max }^{\mu}$ is determined from the fit with a Gaisser-Hillas function as shown in Fig. 8. To reduce the EM contamination of the traces in the WCDs only inclined showers with zenith angles $\theta=\left[55^{\circ} ; 65^{\circ}\right]$ are used in the current analysis. Additionally, a cut on the primary energies $E>20 \mathrm{EeV}$ is applied to have sufficient number of muons for the reconstruction of the profile, and a cut on the station distances to the core $1700 \mathrm{~m}<r<4000 \mathrm{~m}$ ensuring at the same time similar proton and iron selection efficiencies and good $X_{\max }^{\mu}$ resolution. The final data set contains 481 events, the systematic uncertainty on $X_{\max }^{\mu}$ is around $17 \mathrm{~g} / \mathrm{cm}^{2}$, the resolution is $100(80) \mathrm{g} / \mathrm{cm}^{2}$ at $10^{19.3} \mathrm{eV}$ for $p(\mathrm{Fe})$ and $50 \mathrm{~g} / \mathrm{cm}^{2}$ at $10^{20.0} \mathrm{eV}$.

The evolution of the MPD in data and the predictions for protons and iron, obtained with EPOS-LHCand QGSJetII-04, are shown in Fig. 9. Comparison of the measured ER $D_{10}^{\mu}=d\left\langle X_{\max }^{\mu}\right\rangle / d \lg (E / \mathrm{eV})=-25 \pm$ 22 (stat.) \pm 21 (sys.) $\left[\mathrm{g} / \mathrm{cm}^{2} /\right.$ decade $]$ (Fig. 9) with the values for protons $\left(35.9 \pm 1.2\left[\mathrm{~g} / \mathrm{cm}^{2} /\right.\right.$ decade $\left.]\right)$ and iron $\left(48.0 \pm 1.2\left[\mathrm{~g} / \mathrm{cm}^{2} /\right.\right.$ decade] $)$ indicates the transition from lighter to heavier composition with increasing energy. Due to large uncertainties on the absolute values of $\left\langle X_{\max }^{\mu}\right\rangle$ the reconstructed $\langle\ln A\rangle$ strongly depends on the interaction model. For EPOS-LHCthe mass composition is heavier than iron (cf. Figs. 7,9). The deeper $X_{\max }^{\mu}$ for EPOS-LHCis due to a larger elasticity in protonair and pion-air interactions, which results from a better, compared to QGSJetII-04, description of rapidity gap distributions measured at the LHC [16]. Thus the better agreement of $\langle\ln A\rangle$ from $\left\langle X_{\max }^{\mu}\right\rangle$ and $\left\langle X_{\max }\right\rangle$ measurements for QGSJetII-04 (Fig. 7) might be rather accidental.

\section{Muon content of highly inclined showers [17]}

Using highly inclined events (zenith angles $>62^{\circ}$ ) an almost direct measurement of the muon shower content can be performed. The approach consists in production of the reference muon density lateral profiles $\rho_{\mu, 19}(\vec{r} ; \theta, \phi)$ with a shape weakly depending on energy, on the type of the primary nuclei, on the hadronic interaction model and on the software used for shower simulations. For a given muon density $\rho_{\mu}$, a muon scale factor $N_{19}$ with respect to $\rho_{\mu, 19}(\vec{r} ; \theta, \phi)$ is then introduced: $\rho_{\mu}=$ $N_{19} \rho_{\mu, 19}(\vec{r} ; \theta, \phi)$. By construction $N_{19}$ does not depend on the zenith angle. Finally one compares scaling factors in data and in Monte-Carlo (MC) simulations $R_{\mu}=$ $N_{19}^{\text {data }} / N_{19}^{\mathrm{MC}}$.

The production of the reference profiles $\rho_{\mu, 19}(\vec{r} ; \theta, \phi)$ for different zenith $\theta$ and azimuth $\phi$ angles is done using QGSJetII-03 protons at $10^{19} \mathrm{eV}$. Hybrid events with energies above $10^{18.6} \mathrm{eV}(\approx 4 \mathrm{EeV})$, corresponding to $100 \%$ trigger efficiency of the SD, are accepted for the analysis, the zenith angles are limited to the $\left[62^{\circ} ; 80^{\circ}\right]$ range. In total 174 events remain after the selection.

The muon scales $\left\langle R_{\mu}\right\rangle$ in data and simulations for protons and iron are shown in Fig. 10. Similarly to the measurements of $X_{\max }$, risetime asymmetry and MPD, the slope $d\left\langle R_{\mu}\right\rangle / d \lg (E / \mathrm{eV})$ in data differs from the MC predictions for constant compositions and is compatible with a transition to a heavier mass composition. The absolute values of $\left\langle R_{\mu}\right\rangle$ in data correspond to masses close to or heavier than iron, thus being in contradiction with the results on $\langle\ln A\rangle$ from the analysis of $X_{\max }$. Similar conclusions were obtained on the muon content of the vertical showers [18]. To quantify the discrepancy, the mean logarithmic mass from the analysis of $X_{\max }$ distributions can be converted into the mean logarithmic muon scale $\left\langle\ln R_{\mu}\right\rangle$ [17] thus leading to a one-to-one relation between $\left\langle\ln R_{\mu}\right\rangle$ and $\left\langle X_{\max }\right\rangle$. The results presented in Fig. 11 show, that the muon content $\left\langle\ln R_{\mu}\right\rangle$ in data is $(30-80) \%$ larger compared to simulations for primary masses $\langle\ln A\rangle$ corresponding to the measured $\left\langle X_{\max }\right\rangle$ Nevertheless, the minimum difference between data and EPOS-LHCis only $1.4 \sigma_{\text {sys }}$.

\section{Discussion}

The results of studies of the UHECR mass composition at the Pierre Auger Observatory, presented in these proceedings, indicate that $\langle\ln A\rangle$ is not constant for all energies above $10^{17} \mathrm{eV}$. Specifically, the ER of $X_{\max }\left(d\left\langle X_{\max }\right\rangle / d \lg (E / \mathrm{eV})\right)$ shows that the composition is getting lighter below $2 \mathrm{EeV}$ and then heavier for higher energies. Conversion of the moments of $X_{\max }$ distributions to the moments of the logarithmic mass produces results that are consistent with a mixed composition within 

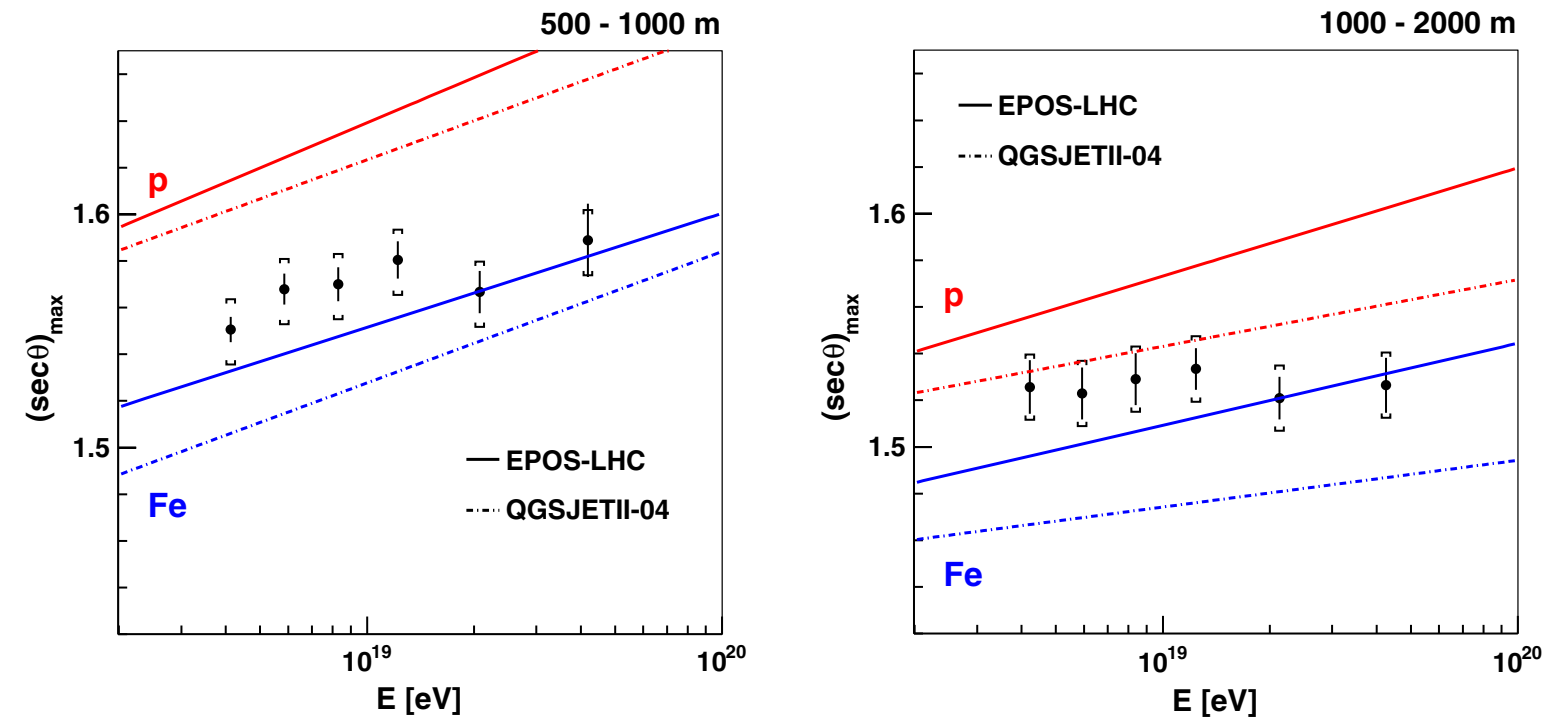

Figure 6. Dependence of $\sec (\theta)_{\max }$ on energy in data (points) and simulations with EPOS-LHCand QGSJetII-04for protons and iron nuclei in the distance ranges $r=500-1000 \mathrm{~m}$ (left) and $r=1000-2000 \mathrm{~m}$ (right).
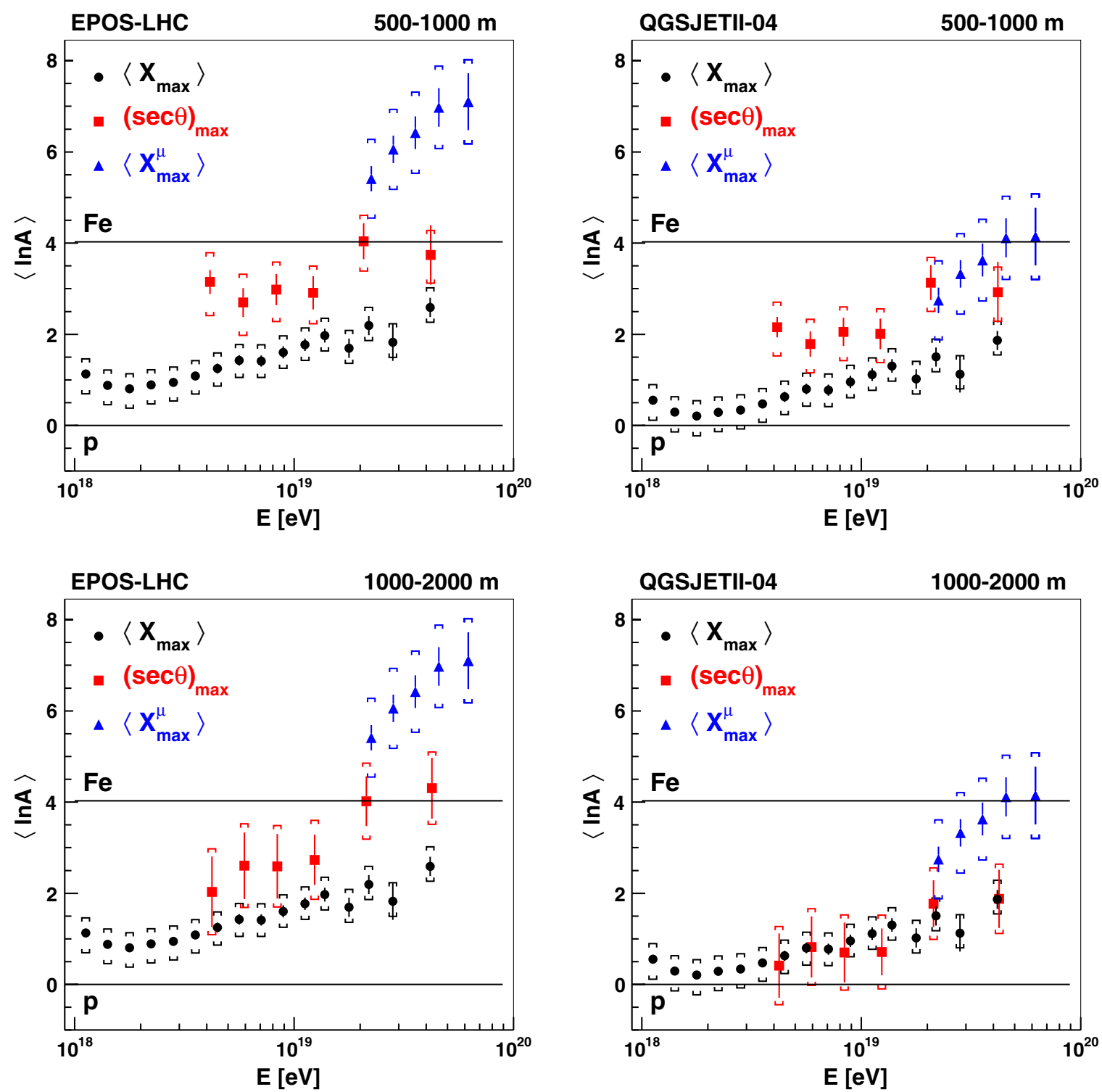

Figure 7. Comparison of the mean logarithmic masses from the measurements of $X_{\max }, \sec (\theta)_{\max }$ and $X_{\max }^{\mu}$. Distance ranges $r=$ $500-1000 \mathrm{~m}$ and $r=1000-2000 \mathrm{~m}$ refer only to the analysis of $\sec (\theta)_{\max }$. 

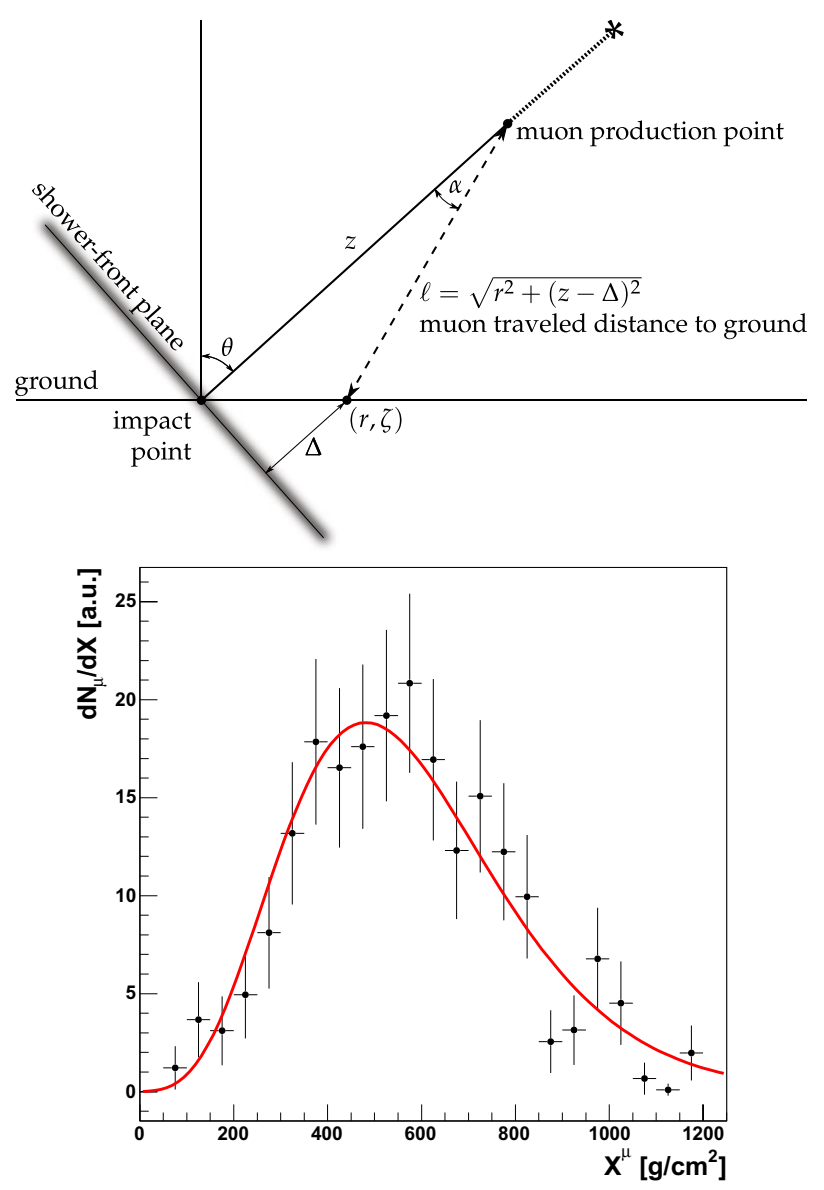

Figure 8. Top: the geometry used for the estimation of the muon production height $z$. Bottom: the muon production depth (MPD) profile of a real event with the energy of $33 \pm 1 \mathrm{EeV}$ fitted with the Gaisser-Hillas function (red line).

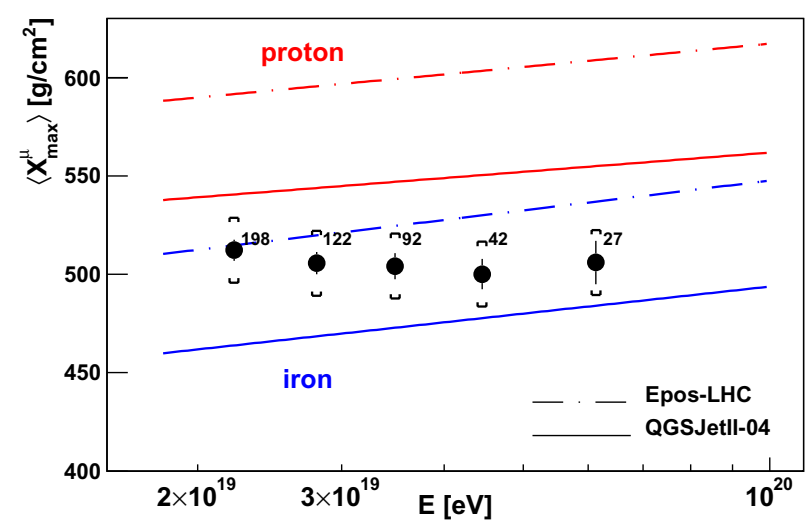

Figure 9. Mean MPD $\left\langle X_{\max }^{\mu}\right\rangle$ in data (points) compared to simulations with EPOS-LHCand QGSJetII-04for protons and iron.

systematic uncertainties when EPOS-LHCis used. For QGSJetII-04the conclusions are less certain and even an unphysical variance of logarithmic masses $\left(\sigma_{\ln A}^{2}<0\right)$ is observed for $E \gtrsim 3 \mathrm{EeV}$, though the values differ from zero by less than $2 \sigma_{\text {stat }}$.

Analysis of the correlation between $X_{\max }$ and $S(1000)$ provides a strong evidence on the presence of several types of nuclei near the ankle $(\approx 5 \mathrm{EeV})$ in the primary spectrum with the spread of the masses $\sigma(\ln A)>1$ for all interaction models used (EPOS-LHC, QGSJetII-04, Sibyll 2.1). The data are described well by mixed

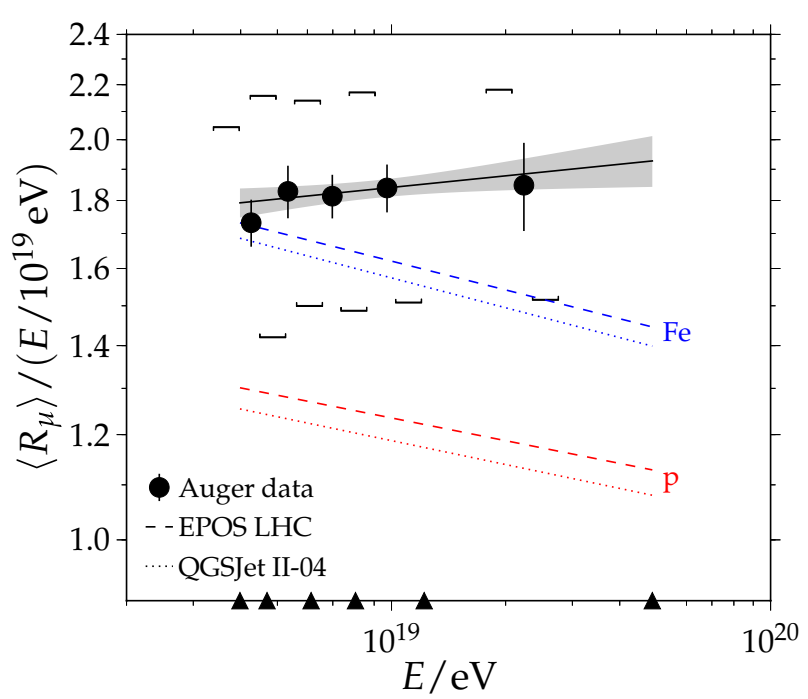

Figure 10. The muon scales $\left\langle R_{\mu}\right\rangle$ in data and in the MC simulations with EPOS-LHCand QGSJetII-04for protons and iron.

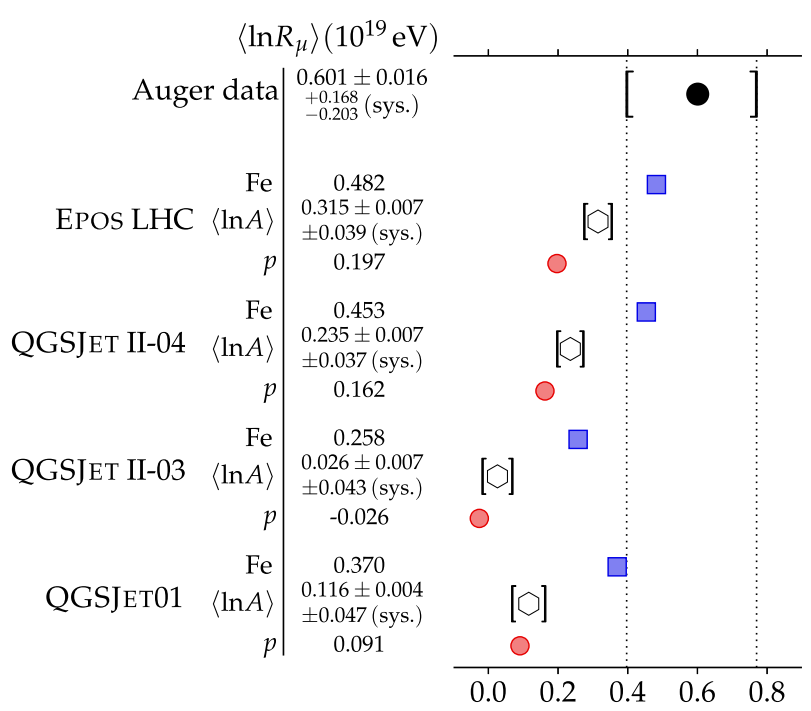

Figure 11. Comparison of $\left\langle\ln R_{\mu}\right\rangle$ at $10^{19} \mathrm{eV}$ in data to the values in simulations for protons, iron and for the mixes with $\langle\ln A\rangle$ from the measurements of $X_{\max }$.

compositions containing nuclei heavier than helium $A>4$. The results are robust with respect to modifications of the key parameters of the interaction models. In [19] the $X_{\max }$ distributions measured at the Pierre Auger Observatory were interpreted in terms of fractions of protons, helium, nitrogen and iron. For QGSJetII-04and Sibyll 2.1the composition was found to be close to $0.5 \mathrm{p}-0.5 \mathrm{He}(\sigma(\ln A) \approx 0.69)$ for energies $\lg (E / \mathrm{eV})=$ 18.5-19.0. The correlation coefficient for $0.5 \mathrm{p}-0.5 \mathrm{He}$ mix for these models is $r_{\mathrm{G}} \approx 0.08$ and is in contradiction with the value found in data, indicating thus that QGSJetII04and Sibyll 2.1do not describe $X_{\max }$ distributions and $\left(X_{\max }, S(1000)\right.$ ) correlation in a self-consistent way. For EPOS-LHCthe fits of $X_{\max }$ distributions produce a mixed composition including protons, helium and nitrogen with $\sigma(\ln A) \approx 1.2$ and $r_{\mathrm{G}} \approx-0.094$ in a better agreement with the correlation analysis.

The ERs for the azimuthal asymmetry in the risetime and for the MPD differ from the ERs for constant 
compositions and are consistent with the increase of $\langle\ln A\rangle$ for the energies $E>20 \mathrm{EeV}$. The risetime asymmetry analysis reveals further possible deficiencies in QGSJetII04leading to an unphysical dependence of the mass composition on the range of the WCDs distances to the shower axis. In its turn, the absolute value of $\left\langle X_{\max }^{\mu}\right\rangle$ is beyond the predictions for iron for EPOS-LHC, corresponding thus to masses $\langle\ln A\rangle>4$.

Similarly to other analyses, the observed gain of the muon scale with energy in inclined showers $d\left\langle R_{\mu}\right\rangle / d \lg (E / \mathrm{eV})$ differs from predictions for constant compositions and indicates an increase of the primary mass for $E>4 \mathrm{EeV}$. Muon content in simulations, corresponding to the primary mass derived from analysis of $X_{\max }$ distributions, is found to be $(30-80) \%$ smaller than in data, but the difference with EPOS-LHCis only at the level of $1.4 \sigma_{\text {sys. }}$. Similar conclusions for the vertical events were obtained in [18].

\section{References}

[1] A. Aab et al. (Pierre Auger), Nucl. Instrum. Meth. A798, 172 (2015), 1502.01323

[2] T. Pierog, I. Karpenko, J.M. Katzy, E. Yatsenko, K. Werner, Phys. Rev. C92, 034906 (2015), 1306.0121

[3] S. Ostapchenko, Phys. Rev. D83, 014018 (2011), 1010.1869

[4] E.J. Ahn, R. Engel, T.K. Gaisser, P. Lipari, T. Stanev, Phys. Rev. D80, 094003 (2009), 0906.4113
[5] A. Porcelli (Pierre Auger), PoS ICRC2015, 420 (2016), 1509.03732

[6] A. Aab et al. (Pierre Auger), Phys. Rev. D90, 122005 (2014), 1409.4809

[7] J. Matthews, Astropart. Phys. 22, 387 (2005)

[8] K.H. Kampert, M. Unger, Astropart. Phys. 35, 660 (2012), 1201.0018

[9] P. Abreu et al. (Pierre Auger), JCAP 1302, 026 (2013), 1301.6637

[10] A. Aab et al. (Pierre Auger), Phys. Lett. B762, 288 (2016), 1609.08567

[11] P. Younk, M. Risse, Astropart. Phys. 35, 807 (2012), 1203.3732

[12] J. Alvarez-Muñiz, R. Engel, T.K. Gaisser et al., Phys. Rev. D66, 033011 (2002), astro-ph/0205302

[13] R. Gideon, R. Hollister, JASA 82, 656 (1987)

[14] A. Aab et al. (Pierre Auger), Phys. Rev. D93, 072006 (2016), 1604.00978

[15] A. Aab et al. (Pierre Auger), Phys. Rev. D90, 012012 (2014), [Erratum: Phys. Rev. D92, no.1, 019903(2015)], 1407.5919

[16] T. Pierog, B. Guiot, K. Werner, PoS ICRC2015, 337 (2016)

[17] A. Aab et al. (Pierre Auger), Phys. Rev. D91, 032003 (2015), [Erratum: Phys. Rev. D91, no. 5, 059901(2015)], 1408.1421

[18] A. Aab et al. (Pierre Auger), Phys. Rev. Lett. 117, 192001 (2016), 1610.08509

[19] A. Aab et al. (Pierre Auger), Phys. Rev. D90, 122006 (2014), 1409.5083 